\title{
Surfaces
}

\section{Le Deuil de la Télévision}

\section{Deborah Esch}

Volume 1, 1991

URI : https://id.erudit.org/iderudit/1065254ar

DOI : https://doi.org/10.7202/1065254ar

Aller au sommaire du numéro

Éditeur(s)

Les Presses de l’Université de Montréal

ISSN

1188-2492 (imprimé)

1200-5320 (numérique)

Découvrir la revue

Citer cet article

Esch, D. (1991). Le Deuil de la Télévision. Surfaces, 1. https://doi.org/10.7202/1065254ar d'utilisation que vous pouvez consulter en ligne.

https://apropos.erudit.org/fr/usagers/politique-dutilisation/ 


\title{
Le Deuil de la Télévision
}

\author{
Deborah Esch
}

La télévision est, à plus d'un titre, affaire de survie. Le feuilleton Knots Landing en donne un bon exemple ou alors il n'est rien[1]. L'histoire de ce feuilleton, qui est resté bien longtemps à l'affiche, commence de manière malencontreuse mais symptomatique puisque son lancement fut différé. En effet, alors que l'idée de ce programme avait été initialement élaborée en 1977, c'est-à-dire avant l'avènement du feuilleton mélodramatique en heures de haute écoute (et de sa prolifération avec Dynastie, l'Empire des Colby, Falconcrest et Flamingo Road ), sa focalisation explicite sur la classe

moyenne éveilla les réserves des programmateurs de CBS. Selon David Jacobs, le créateur de Knots Landing et co-producteur exécutif avec Michael Filerman de ce programme, le réseau CBS s'avéra être hésitant devant le projet, et demanda "quelque chose du même ordre mais plus clinquant, plus sensationnel, quelque chose dont la promotion serait plus facile: plus une épopée qu'un feuilleton" [2]. Ce qu'il obtint. Ce fut Dallas qui correspondit à ses exigences, et c'est sur la base du succès de ce programme que l'idée de Knots Landing fut récupérée "d'un fond de tiroir" et lancée à partir du feuilleton à grand succès qui l'avait supplanté pour des raisons d'ordre idéologique dans sa phase conceptuelle. Jacobs ajoute que "en réalité, Knots Landing a toujours été mal adapté au caractère d'un Dallas. L'agressivité de sa dominante de classe (moyenne) a toujours fait obstacle". C'est partiellement pour cette raison, continue à suggérer Jacobs, que Knots Landing eut du mal à joindre les deux bouts pendant un certain temps. Sans connaître l'échec, il fut néanmoins étranger au succès; il continua cependant à survivre, sans identité propre, soit ignoré par la presse soit perçu comme le petit frère remorqué par Dallas[3]. Et cependant, il a fait ce qu'il devait faire pour continuer; il pouvait survivre[4]. Ce que le programme dut faire, surtout pour résister à une programmation qui le plaçait en face de l'agressif Hill Street Blues, "le programme de télévision jouissant de la meilleure réception critique en son temps", c'est, toujours selon Jacobs, "de raconter des histoires de classe bien autre que moyenne"[5]. Mais Knots Landing

incarne aussi un exemple d'un autre ordre, celui de la survie à la télévision et de la télévision, qui n'est pas l'enjeu des luttes concurrentielles pour les taux d'écoute mais plutôt de certaines des conditions de possibilité et d'impossibilité du médium lui-même.

Cette affirmation trouverait sa référence dans les 200ème et 201ème épisodes ("Noises Everywhere" ("Des bruits partout"), première et seconde 
parties), qui furent l'objet d'une attention considérable dans la presse lors de leurs diffusions les 3 et 10 décembre 1987 à cause de leur caractère expérimental. Dans ce que TV Guide qualifia d' "entreprise hors du commun et pleine de risques", les acteurs furent appelés à improviser pendant deux jours alors qu'une équipe de son et quatre caméras enregistraient seize heures de vidéo, fournissant ainsi la base du scénario qui allait suivre[6]. Au cours de cet exercice que le co-producteur Larry Kasha compara à "une visite chez le psychanalyste", les personnages écrivirent leur histoire avant de la jouer[7].

Les épisodes en question tournent autour des funérailles de Laura Murphy Avery Sumner, depuis longtemps membre de cette communauté de la Californie du sud, que son ex-mari Richard Avery (joué par John Pleshette, acteur occasionnel et réalisateur) qualifie sardoniquement, à l'occasion de ces funérailles et après avoir été absent du programme plus de quatre ans, de "véritable utopie, la communauté idéale du système solaire". Cette communauté est fondée sur la métonymie puisqu'elle consiste en lotissements adjacents dans un développement immobilier. En outre, cette métonymie spatiale trouve son homologue temporel dans la succession narrative caractéristique du format du feuilleton scandé par "une maudite chose après une autre". C'est aussi une communauté dont l'histoire, telle qu'elle est relatée dans Knots Landing: The Saga of Seaview Circle, est celle d'une lutte continue pour survivre: "Comme les autochtones américains qui vécurent ici il y a plus de deux cent ans, y lisons-nous, les [familles de voisins] ont placé leur confiance dans l'intimité communautaire comme meilleure défense contre les dangers du monde extérieur. En ce qui concerne les autochtones, il s'est avéré que leur mode de vie ne sut résister aux pressions de l'extérieur ["les missionnaires apportèrent, avec leur culture et leur technologie, la variole et les maladies vénériennes, et leur village fut presqu'entièrement anéanti]; quant aux voisins de Seaview Circle, ce mode de vie est en train de subir l'épreuve du temps."[8]Dans les épisodes qui ont immédiatement précédé la veillée et les funérailles jouées en improvisation, Laura

(Constance McCashin) échoue contre l'épreuve du temps imposée au cul-desac[9] elle se heurte à sa propre fin en apprenant qu'elle est condamnée par un cancer cérébral. (Il n'est pas superflu de noter ici que pendant près de la moitié des dix années du programme Laura a fait l'expérience de la mort vivante, celle de la ménagère opprimée [selon ses propres termes], une vie dont la mémoire a été inscrite dans les séquelles de cette autre mort.)[10]. Prenant une décision que les membres de sa famille et ses amis perçoivent comme une violation du contrat social qui sous-tend cette communauté étroitement unie, Laura se résout à partir et à mourir seule dans une clinique du Minnesota, loin des siens alors qu'"elle aurait fort bien pu mourir ici"[11]comme le clame Richard

dans un moment de fureur récriminatoire. Cet "ici" est bien entendu doublement déictique, désignant à la fois le ici à Knots Landing et le ici sur Knots Landing. Car la décision vexante de Laura entraîne aussi une violation apparente du contrat diégétique puisque le spectateur se voit privé de l'événement-pivot de sa mort qui ne s'inscrit que par le rapport d'une tierce 
instance, son mari, Greg Sumner, relatant un appel téléphonique de la clinique. Aux prévisibles questions de ceux qui veulent savoir si Laura avait beaucoup souffert vers la fin, le taciturne Sumner (William Devane) ne peut répondre que "Je n'en sais rien, je n'y étais pas". Ce qui est encore plus pertinent c'est que la mort de Laura in absentia, hors champ, prive à la fois les personnages et nous les spectateurs d'un des rites nourriciers de l'univers des feuilletons, à savoir le fait que la mort donne l'occasion aux vivants, aux survivants, d'adresser la parole aux morts, de dire ce que pour une raison ou pour une autre ils n'ont pu ou n'ont pas voulu dire quand cela pouvait être entendu et compris, quand cela pouvait entraîner une riposte. Habituellement l'apostrophe rituelle au mort a une double fonction: elle réaffirme les valeurs prédominantes de la famille et d'une certaine communauté, et elle effectue une réconciliation provisoire des différences passées sous la forme d'une ouverture sur l'avenir projeté dans la succession sérielle. Le geste radical de réécrire cette convention nous met ici

face à un vide: il n'y a pas de corps, de dépouille à apostropher[12]; la dépouille de Laura, nous apprend-on, est censé arriver par air cargo. La veillée se prolonge lorsque le vol est retardé et que les restes de Laura sont livrés par erreur à une autre entreprise de pompes funèbres. C'est donc une Laura spectrale et désincarnée qui revient s'adresser à ceux qui lui ont survécu, une Laura dont le médium est la vidéocassette[13].

esch-1 esch-2 esch-3 esch-4 esch-5 esch-6 esch-7 esch-8 esch-9

La scène du deuil est donc déjà hantée par le revenant qui élit résidence dans le magnétoscope. Le fantôme dans cette machine ne se laisse pas conjuré puisque lui-même conjure, exerçant son contrôle sur les survivants à partir d'un autre temps et d'un autre lieu. Et la figure, la fiction de l'apostrophe renversée d'avant et d'outre tombe, interrompt de manière fatale le système spéculaire de la représentation mimétique sur laquelle ce programme, comme tous ceux du genre, est fondé. Tout ceci exige une analyse un peu plus

poussée, un relâchement des fils enchevêtrés du n_ud, et en particulier ceux de la temporalité et de la technologie, de la subjectivité et de la différence sexuelle, de la rhétorique et de la représentation.

L'annonce préliminaire de Sumner aux participants de la veillée funéraire, que "Laura a laissé des messages d'adieux sur une bande magnéto; je vais aller la passer dans le petit salon", déconcerte et provoque un sentiment de malaise. Celui-ci se reflète dans la question posée par Richard (qui a des craintes à avoir puisque, dans un épisode antérieur, il avait, par la force des armes, pris Laura en otage dans son propre salon et l'avait ensuite abandonnée avec leurs deux enfants): "Quelle sorte de messages?". Question à laquelle Sumner répond avec désinvolture: "Te préoccupes pas, tu y survivras", réponse qui est loin de constituer une garantie. Celle-ci d'ailleurs est ironisée dans les images qui suivent où on le voit appuyé au mur du petit salon près de sa collection d'armes à feu, l'une d'elles pointée directement vers sa tête. Dans quel sens survivent-ils, ou non, à cette scène? Et nous? 
Que la "rhétorique [de l'apostrophe] n'ait rien d'un consolant simulacre" devient évident dans ce qui arrive lorsque Laura s'adresse à ceux qui lui survivent, les uns après les autres[14] Les femmes, Val (Joan Van ArK), Abby (Donna Mills) et Karen (Michelle Lee) paraissent être ensorcelées. Ayant perdu la parole, elles ne peuvent que produire des rires inarticulés et des larmes. Il serait possible même de leur attribuer une espèce de reconnaissance muette au fait que Laura ait irrévocablement disparu, qu'elle ne puisse rien savoir de ce qui se passe dans leur mémoire. En termes de rhétorique, ce qui confère le pouvoir de la parole à l'absent, au

mort, c'est-à-dire la figure de la prosopopée[15], semble, par effet de symétrie, assurer que les survivants soient frappés de mutisme, saisis dans un photogramme fatal. Les limites de leur subjectivité en tant que spectateurs sont marquées alors même que cette subjectivité est constituée dans et par l'allocution, qui est à la fois sa condition de possibilité et d'impossibilité. C'est comme si Laura disait non pas "Tu survivras" ou même "Tu survis", mais "Tu es mort. Ce sont tes funérailles à toi car tu n'as rien à dire et bien des comptes à rendre".

Dans les figures muettes de Val, d'Abby, et de Karen, le spectateur est simultanément responsabilisé et interdit de réponse[16]. Cette simultanéité est elle-même l'effet d'une structure temporelle plus compliquée, celle du pré-enregistrement par l'entremise duquel la morte dicte à l'avance ce que ses survivants font ici et maintenant. Dans cet exemple précis, l'ici et maintenant de l'instant enregistré, l'instant de l'enregistrement, est coupé du présent du visionnement par une mort qui fait de ce passé un passé absolu, son antériorité déterminante mais irrévocable[17]. Si la mort est bien le thème de cet épisode (ce qui est suggéré par la question de Sumner à Jill Bennett [Teri Austen], "La partie te plaît?", et la réplique de cette dernière,

"Tu pourrais changer de thème la prochaine fois"), elle a aussi une fonction syntaxique: elle marque une rupture, une disjonction (dans le temps) qui n'est pas disponible en tant qu'événement et qui est impensable en tant que présent. C'est cette non-disponibilité qui est figurée dans la "décision" de Laura de partir mourir ailleurs et dans la perplexité et les résistances qu'elle provoque. Les réactions assourdies au point d'être silencieuses des femmes présentes à la veillée indiquent que le passé du pré-enregistré, du déjà écrit, ne peut être assimilé au présent, ne peut être approprié ni comme cognition (par le biais d'une réinscription de cette tropologie du sujet dans un mode spéculaire de connaissance) ni comme mémoire (sur le modèle hégélien ou freudien du souvenir comme Erinnerung idéalisante et internalisante)[18]. Car ce qui est en question, c'est un passé qui n'a jamais été présent, qui résiste à la clôture de la mémoire intériorisante, qui "ne se laissera jamais ranimer dans l'intériorité d'une conscience[19]. Le predicament [mot que Derrida juge intraduisible, cf. Psyché p. 28] du sujet visionneur consiste en ce que l'autre, l'autre en tant qu'autre qui peut mourir, aura toujours déjà parlé, aura eu le premier mot. De fait, le sujet est sommé de répondre mais ne peut pas, il est déjà porteur d'une dette dont il ne pourra s'acquitter (une 
condition dont les requêtes et les legs de Laura envers Karen sont la figuration)[20].

Contrairement aux autres survivants, Sumner répond au message d'adieu de sa femme (qu'il visionne en privé après s'être retiré) en cherchant à entraîner son image vidéo dans une espèce de dialogue qui serait la duplication d'échanges caractéristiques à la Hepburn et Tracy (Laura: "Tu as été le seul à me donner le sentiment d'être vraiment aimée. Bien sûr tu t'y prenais d'une manière bien bizarre mais ça marchait"; Greg: "Je riais à tes blagues insipides"). Ce mode de réponse figure un refus de sa responsabilité, une résistance à la dette qu'il a envers l'autre, une résistance à la priorité et à la fin de non-recevoir de celle-ci. Face à cette divergence par rapport à la reconnaissance muette des femmes dans la scène précédente, il peut être tentant d'invoquer dans ce contexte une subjectivité qui serait spécifiquement masculine et dont la caractéristique serait un effort de domestication de l'altérité de l'autre, de son appropriation en tant que sens, de sa conception de l'autre en tant que l'autre d'un soi, d'un moi, de moi-même ("Pourquoi m'as-tu laissé seul? Je n'arrive pas à comprendre pourquoi tu m'as laissé tout seul.", dit Greg, alors que l'image de Laura refuse à s'engager dans le dialogue qu'il tente de construire. Il commence alors à perdre contrôle et de la situation et de lui-même. Ou encore, ses paroles d'adieu: "Je t'aime. J'espère que je ne finirai pas par te haïr".), même si l'on pourrait arguer la mort de l'autre comme ce qui constitue la relation du soi à lui-même, cette relation que nous appelons subjectivité "de première instance". L'asymétrie d'un passage impossible (entre les morts et les vivants, entre alors et maintenant) reflétée dans l'asymétrie de la structure des prises de vue en champ/contre-champ dans la scène qui enregistre cet échange sans issue, débouche sur la non-compréhension ("Tu me laisses dans le pétrin, Red. Je ne sais pas ce que tu cherches à me dire."), résultat de la tentation de négocier ce qui n'est pas négociable, de mettre en dialogue l'hétérogénéité radicale qui comprend le ]predicament irréductible du sujet visionnant. L'inscription de la différence sexuelle en ce lieu est rendue encore plus compliquée par le fait que la ligne de démarcation des sexes dans Knots Landing (programme qui selon Ted Shackelford, l'acteur qui joue Gary Ewing, est "centré sur les femmes")[21]est instable,

volatile, comme des "test patterns", s'entrecroisant et se recoupant pour s'enchevêtrer dans des noeuds inextricables [22]. A le dire trop rapidement et grossièrement, la mystification n'est pas complète: le sujet masculin endeuillé se trompe mais pas entièrement; en un sens il a raison aussi. Le mouvement qui va du "Voyons ce que tu as à dire" quand il enclenche le magnétoscope jusqu'au "Comme tu veux, (si tu le dis)" quand il commence à s'affaisser, le réduit à hocher la tête en silence et dans les larmes, et trace l'impossibilité de faire parler l'autre sans que l'autre ait déjà parlé, ait dit quelque chose d'autre que ce qui se donne à voir, lire et comprendre. L'autre parle autrement, dans une allégorie qui peut être généralisable comme l'allégorie de la télévision.

"Ce qui est propre à la télévision - la possibilité de diffuser en direct, la production électronique de l'image dans le présent- devient 
le terme de son imaginaire exploité, le phantasme généralisé [...] selon lequel l'image est en direct, et dirigée vers moi [...] et ce phantasme [...] est alors pris pour la réalité fondamentale de la télévision et de ses programmes."[23]Ce qui est peut-être ironique dans le cas de Knots Landing c'est qu'une certaine résistance à ce phantasme généralisé se dévoile dans l'usine aux phantasmes de la télévision des séries mélodramatiques, qui démystifie alors même qu'elle exploite cet imaginaire. De même, la série se base sur un mode de représentation mimétique et cependant, dans les scènes comme celle où l'image de Laura s'adresse aux veilleurs et les implique, elle offre un lieu de résistance à ses séductions faciles. Jacobs, et ses inconditionnels, ont donc dans cette mesure raison de revendiquer une "différence" pour Knots Landing par rapport aux autres feuilletons développés après 1979. Mais cette différence ne résiderait pas dans sa focalisation caractéristique sur le monde de la classe moyenne qui s'est évaporée pendant le cours de l'histoire du programme. Sa "différence" serait plutôt fonction de l'adéquation relative de sa réponse "à la question que tout le monde pose [...] de savoir comment introduire de la résistance dans cette industrie culturelle. Je pense que la seule ligne à suivre est de produire des programmes pour la TV ou quoi que ce soit qui produise chez le spectateur ou le client en général un effet d'incertitude et de trouble [...]. On ne peut introduire des concepts, on ne peut introduire une argumentation. Ce genre de média n'est pas le lieu de ce genre de choses, mais on peut introduire un sentiment de trouble dans l'espoir que ce trouble sera suivi par la réflexion."[24]

La possibilité que l'allégorie télé-épitaphique, relayée par la troublante apostrophe par vidéo de Laura, hante toute la télévision et ,en particulier et surtout, la télévision en direct, est figurée par anticipation dans la première partie de cet épisode, dans la scène où Mack Mackenzie (Kevin Dobson) cherche les mots capables de consoler son vieil ami Sumner. L'expression de la sympathie trouve sa forme dans une citation qui est au bord du cliché: "A quoi bon être irlandais, hein?". Et devant le "Quoi" de non-compréhension de Sumner, un quoi interrogatif qui scande le récit de Knots Landing à intervalles réguliers, Mack élabore: "Je crois qu'il ne sert à rien d'être irlandais si tu ne sais pas qu'un jour le monde te fendra le coeur". La réponse de Greg, "Si tu le dis", anticipe le "Si tu le dis " qu'il lance plus tard à l'image vidéo de Laura, et oblige Mack à donner la source de sa citation jusqu'alors non-identifiée: "C'est ce que Daniel Patrick Moynihan a dit après que Kennedy ait été tué.". Et Sumner de répondre: "On dirait qu'il n'attendait que ça.". Mack: "Quoi?". Sumner: "Il attendait qu'on lui fende le coeur"[25].

Toute une avalanche de programmes de 25ème anniversaire et de rediffusion nous l'a rappelé récemment: l'assassinat de Kennedy et ses funérailles sont devenues pour toute une génération de spectateurs (et pas seulement américains) le paradigme de l'événement télévisé rapporté en direct dans nos salons. L'idée que Moynihan, et nous avec lui, n'attendait que ça, confirme que la possibilité de la mort, la mort de l'autre, "a lieu" pour ainsi dire avant l'événement, dans un passé qui n'aura jamais existé 
dans le présent d'une diffusion en direct, et cela d'une manière qui nous marque par anticipation en tant que sujets. Les funérailles, de Laura,

de John Kennedy, mettent en scène le deuil auquel le discours télévisuel, et la subjectivité spectatrice, ne peuvent échapper, mais auquel ils parviennent, plus ou moins, à survivre[26].

\section{Deborah Esch \\ University of Toronto}

Dept. of English

Toronto - ONT M5S 1A1 - Canada

Surface Page d'Acceuil/Home Page

]

[1] Qu'il ne soit rien s'il n'en est pas l'exemple est suggéré par le rôle-pivot joué par le "O" de "Knots" dans une des versions du générique visuel du feuilleton, dans lequel le " $\mathrm{O}$ " est bien la première lettre à se manifester sur l'écran, ligne courbe se refermant sur elle-même figurant le cul-se-sac (Seaview Circle), qui servit de lieu de l'action surtout au cours des premières années du feuilleton. Ce "O" se donne bien entendu aussi à lire en tant que zéro arithmétique, lieu-tenant hétérogène dans l'ordre des nombres, ainsi que chiffre asémantique. Le titre d'un des épisodes, par ailleurs emprunté au répertoire de chansons de Lilimae Clements, "Will the Circle Be Unbroken?" ("Le Cercle sera-t-il maintenu?"), pose une question dont la réponse est certainement négative: le cul-de-sac est toujours déjà un cercle rompu, son intérieur s'ouvrant sur un dehors qui met en danger son intégrité en tant qu'intériorité (ce qui se reflète dans la bien trop prévisible thématique de ceux-d'ici/ceux du dehors qui anime la communauté de Knots Landing.). Cette mise en espace figure la résistance de la forme feuilleton à la clôture narrative et à la totalisation, aussi bien que la résistance de l'épisode singulier à toute réinscription non-problématisée dans la succession sérielle énumérative du feuilleton. A propos de la frustration de la clôture dans les feuilletons et les mélodrames, cf. par ex. Jeremy Butler, "Notes on the Soap Opera Apparatus: Televisual Style and As the World Turns" in, Cinema Journal, 25: 3 (Printemps 1986), ainsi que Sandy Flitterman Lewis, "All's Well That Doesn't End - Soap Opera and the Marriage Motif", in Camera obscura, 16 (janvier 1988), pp.119-127.

[2] David Jacobs, "Introduction" à Laura Van Wormer, Knots Landing: The Saga of Seaview Circle, NY: Doubleday, 1986.

[3] Cette figuration fait allusion au statut de Gary Ewing, le petit frère initialement placé à Dallas, ensuite transplanté avec sa femme Valene pour devenir l'un des quatre couples du cul-de-sac de Knots Landing.

[4] Van Wormer, viii. 
[5] Ibid.

[6] Susan Littwin, "Flying without a net on Knots", in TV Guide, 11: 48, 569 (28 novembre 1987), p.6.

[7] Littwin écrit qu'au cours de l'improvisation qui donna aux acteurs l'occasion d'écrire leurs propres histoires, "parfois ils essayaient d'élargir leurs rôles, parfois de rendre leurs personnages plus aimables. Mais la plupart du temps ils essayaient de rendre ces personnages davantage à leur propre mesure. Ce qui se révéla dans l'improvisation c'est la relation du moi et de son ombre qui se développe entre un acteur et son personnage dans un feuilleton de longue durée. "Je veux faire davantage avec ma vie", me dit [Kevin] Dobson un jour sur le plateau de tournage. "Vous parlez de Kevin ou de Mack [Mackenzie]?", demandai-je. Il haussa les épaules. "Ce doit être les deux, ils sont interchangeables"." (p.7). Une telle identification (fondée sur le postulat de l'interchangeabilité) se produit aussi souvent du côté du spectateur comme effet du mode de représentation mimétique propre aux feuilletons mélodramatiques à la télévision .

[8] Van Wormer, 3, 6. Au cours de l'année 1988-89, la maladie vénérienne qui fut un fléau pour les autochtones selon cette histoire est de nouveau diagnostiquée à Knots Landing, cette fois-ci en tant que pièce à conviction dans l'enquête qui fait suite à l'accusation de Val selon laquelle Jill Bennett aurait tenté de l'assassiner. La technologie, la maladie et la mort sont représentées comme indissociables dans le feuilleton.

[9] Ce que nous appellerons dorénavant cul-de-sac est l'organisation résidentielle en demi-cercle du Seaview Circle de Knots Landing.

[10] La biographie imaginaire de Laura (dans Knots Landing: The Saga of Seaview Circle, publiée en 1986, bien avant départ de McCashin) raconte que "lorsqu'elle avait onze ans, on diagnostiqua un cancer cérébral chez sa mère. Laura dut soudain jouer un rôle de premier plan dans la maison. Elle prit sur elle les obligations ménagères de sa mère sans rechigner [...]. Les soins qu'elle prodiga à cette dernière lui donnèrent un sentiment de valeur, d'importance et de détermination. La mort de sa mère l'année suivante l'anéantit [...]. Le choc devint douleur et enfin colère. Laissée seule avec le cercueil avant l'enterrement, Laura, le visage baigné de larmes, dit "Je te hais, maman, de m'avoir fait ceci." (149-50) Van Wormer scénarise, avant la lettre, un événement de l'histoire de Laura qui précède Knots Landing [ auquel l'épisode de sa mort renvoie allégoriquement.

[11] La base métonymique des relations "intimes" parmi les habitants du culde-sac assure qu'il n'existe au fond pas de distinction entre voisins et amis (ou ennemis). Ces relations fondées sur la métonymie s'entrecroisent avec toute une métaphore de la famille, une généalogie fondée sur la ressemblance (des enfants au père, comme celle invoquée dans la tentative de Gary de prouver la paternité des enfants-jumeaux de Val; de frère à frère, comme dans la revendication par Peter Hollister du titre de frère de Sumner alors qu'il est celui de Jill Bennett. L'indifférence de Sumner quant à la "véracité" de la revendication de Peter et sa disposition à agir comme si Peter était vraiment son frère et à exploiter cette relation tout en ignorant 
son bien-fondé, sont caractéristiques de l'outsider [tel qu'il est défini par Van Wormer, vi].

[12] Comme il n'y a pas de preuve physique de la mort de Laura, la possibilité de son retour futur reste ouverte (les derniers mots de son enregistrement destiné à Greg sont: "A plus tard, ... peut-être."). Un contrepoint d'un certain humour noir à cette absence de preuve de sa mort nous est fourni par le fait qu'une partie de l'action au cours de la veillée funéraire tourne autour de l'urne sur la table du salon de Sumner qui s'avère contenir les cendres de Peter Hollister (que nous avons vu empalé sur un essieu et traîné tout ensanglanté par toute la ville au cours de l'épisode où lui est mort). La résurrection des morts apparents est bien entendu un trait régulier des feuilletons télévisés.

[13] L'apostrophe posthume de Laura à ses survivants rappelle la bande enregistrée laissée à l'intention de Karen par Sid Fairgate, son premier mari, qui est mort sur la table d'opération sans avoir eu l'occasion de lui parler une dernière fois, de la rassurer: "Quoiqu'il m'arrive, mon amour pour toi ne mourra jamais.". Le spectateur est en mesure d'apprécier le progrès technologique entre la diffusion de cet épisode en 1981 et celle de "Bruits partout" en 1987.

[14] Jacques Derrida, Mémoires pour Paul de Man, Paris: Galilée, 1988, p. 50 .

[15] Sur la prosopopée en tant que "fiction d'une apostrophe à un être absent, défunt ou privé de voix, qui pose la possibilité d'une réplique de la part de celui-ci et lui confère le pouvoir de la parole", voir Paul de Man, "Autobiography as De-facement" dans The Rhetoric of Romanticism, New York: Columbia University Press, 1984, pp. 75-76.

[16] La réponse de Richard à l'offre de Laura (et à sa requête) de lui pardonner est un pathétique <<Je l'ai échappé belle $>>$ avec lequel il tente de se dégager de son obligation envers sa mémoire.

[17] Cf. Paul de Man, "Hypogram and Inscription" dans The Resistance to Theory, Minneapolis: University of Minnesota Press, 1986, p.51.

[18] cf. Derrida, 65.

[19] Derrida, p.7.

[20] Laura demande à Karen en tant que voisine, amie et figure de femme exemplaire dans la communauté, d'agir comme mère de remplacement auprès de son enfant Meg. Son legs qui consiste en ce que Karen ait le droit de vendre la maison de Laura sise à côté de la sienne à qui elle veut et au prix qu'elle veut, ce qui revient en fait à choisir ses nouveaux voisins, inscrit le fondement métonymique des relations du cul-de-sac dans l'immobilier.

[21] Littwin, p. 10. "Il est cependant possible que Shackelford, qui est doté d'une belle intelligence et d'une égale capacité d'expression, se sente résigné aux choses comme elles sont dans Knots Landing. Il pense que Gary est trop faible et trop enclin à se trouver pitoyable. "Il devrait faire sa vie, 
avoir du succès dans les affaires comme son frère (le J.R. Ewing de Dallas. Mais sur le plan dramatique cela ne risque guère d'arriver puisque le programme est centré sur les femmes."

[22] Cf. Jacqueline Rose, "Jeffrey Mason and Alice James", dans ]Oxford Literary Review, 8: 1-2 (Numéro spécial sur "Sexual Difference"), 1986, p. 191: "Si ce scénario a quelque importance c'est précisément parce que, aussitôt que l'on dépasse le fait immédiat de la différence sexuelle, on a à faire face à un /pp 16-17/ tel brouillage des démarcations, de la cause et de l'effet [...] un brouillage qui frappe au c_ur de ce que veut dire connaître un corps, connaître la distinction psychique et sexuelle, connaître la limite même du réel." En ce qui concerne la figuration du n ud, de la structure[, voir J. Derrida, Negotiations, ed. Thomas Keenan and Deborah Esch, Minneapolis: University of Minnesota Press, à paraître en 1990.

[23] Stephen Heath et William Skirrow, "Television: A World in Action", in Screen, 18: 2 (été 1977). Voir aussi à ce propos John Wyver, "Television and Postmodernism", in ICA Documents, 4 (Postmodernism), 1986, pp. 52-54.

[24] Jean-François Lyotard, "Brief RÉFLECTIONS on Popular Culture", dans ]ICA Documents, 4 (Postmodernism), 1986, p.58.

[25] Mack, qui jusqu'à sa démission récente assurait le poste de procureur spécial auprès du gouverneur de la Californie, donnait une place de choix à une photo encadrée grand format de John F. Kennedy dans son bureau.

[26] Il est possible bien sûr que ce ne soit là encore qu'une autre manière d'approprier, de récupérer l'antériorité de l'événement en tant que savoir, mémoire, sens, ou, pis encore, comme le pathos de la télévision. Mais le pathos de ce discours télévisé particulier ne peut longtemps survivre à la grammaire de la succession sérielle. Quand Laura demande à Greg: "Et maintenant, quoi? Qu'est-ce qui s'en vient?", l'échange pseudo-dialogique se disloque. Greg est réduit à hocher la tête en signe d'assentiment et c'est Laura qui répond à sa propre question: "Rien, sans doute, je sais que c'est ce que tu dirais. Tu dirais: rien. Moi, je n'en suis pas si sûre que ça.>>. La réponse programmée dans le format sériel est bien entendu: $<<$ La semaine prochaine , sur ]Knots Landing[ ...". Et ce qui arrive dans la foulée de la mort de Laura et de son apostrophe d'outre-tombe n'est guère surprenant: Greg fait une déclaration de mariage à la rivale de Laura, Abby, et a recours à une bande vidéo préenregistrée et faisant sa promotion à la manière de Dating Game pour la convaincre de répondre par l'affirmative. 